Research Paper

\title{
Comparison of HER2 and Lauren Classification between Biopsy and Surgical Resection Samples, Primary and Metastatic Samples of Gastric Cancer
}

\author{
Miao-Zhen Qiu ${ }^{1 *}$, Si-Mei Shi ${ }^{1}{ }^{2 *}$, Min Chen ${ }^{2}{ }^{3 *}$, Jing Wang ${ }^{4}$, Qi-Nian Wu ${ }^{5}$, Hui Sheng ${ }^{5}$, Hui-Zhong Zhang,
} Jing-Ping Yun ${ }^{6}$, Zhi-Wei Zhou ${ }^{7}$, Feng-Hua Wang ${ }^{1 凶}$, Da-Jun Yang ${ }^{5 凶}$, Rui-Hua $\mathrm{Xu}^{1 \bowtie}$

1. Department of Medical Oncology, Sun Yat-Sen University Cancer Center; State Key Laboratory of Oncology in South China; Collaborative Innovation Center for Cancer Medicine, Guangzhou, China;

2. Department of Nursing, Sun Yat-Sen University Cancer Center; State Key Laboratory of Oncology in South China; Collaborative Innovation Center for Cancer Medicine, Guangzhou, China;

3. Department of Nasopharyngeal Carcinoma, Sun Yat-Sen University Cancer Center; State Key Laboratory of Oncology in South China; Collaborative Innovation Center for Cancer Medicine, Guangzhou, China;

4. Department of Pharmacy, The First Affiliated Hospital, Sun Yat-sen University, Guangzhou, China;

5. Department of Experimental Research, Sun Yat-Sen University Cancer Center; State Key Laboratory of Oncology in South China; Collaborative Innovation Center for Cancer Medicine, Guangzhou, China.;

6. Department of Pathology, Sun Yat-Sen University Cancer Center; State Key Laboratory of Oncology in South China; Collaborative Innovation Center for Cancer Medicine, Guangzhou, China;

7. Department of Gastric Surgery, Sun Yat-Sen University Cancer Center; State Key Laboratory of Oncology in South China; Collaborative Innovation Center for Cancer Medicine, Guangzhou, China.

* Miao-Zhen Qiu, Si-Mei Shi and Min Chen contributed equally to the manuscript.

$\triangle$ Corresponding authors: Dr. Feng-Hua Wang, Department of Medical Oncology, Sun Yat-Sen University Cancer Center; State Key Laboratory of Oncology in South China; Collaborative Innovation Center for Cancer Medicine, 651 Dongfeng Road East, Guangzhou 510060, China; Tel.: +86 2087342490 Fax: +86 208734 2490 E-mail: wangfh@sysucc.org.cn Dajun Yang, MD, PhD, Department of Experimental Research, Sun Yat-Sen University Cancer Center; State Key Laboratory of Oncology in South China; Collaborative Innovation Center for Cancer Medicine, 651 Dongfeng Road East, Guangzhou 510060, China; Tel.: +86 2087342285 Fax: +86 2087342285 E-mail: yangdj@sysucc.org.cn Dr. Rui-Hua Xu, Department of Medical Oncology, Sun Yat-Sen University Cancer Center; State Key Laboratory of Oncology in South China; Collaborative Innovation Center for Cancer Medicine, 651 Dongfeng Road East, Guangzhou 510060, China; Tel: +86-20-8734 3333 Fax: +86-20-8734 3295

(1) Ivyspring International Publisher. This is an open access article distributed under the terms of the Creative Commons Attribution (CC BY-NC) license (https://creativecommons.org/licenses/by-nc/4.0/). See http://ivyspring.com/terms for full terms and conditions.

Received: 2017.03.08; Accepted: 2017.06.01; Published: 2017.09.30

\begin{abstract}
Purpose: Patients with advanced or metastatic adenocarcinoma of the stomach or esophagogastric junction for whom trastuzumab therapy is being considered, assessment for tumor human epidermal growth factor receptor-2 (HER2) status is necessary. Can the HER2 status and Lauren classification of the biopsy sample truly represent the HER2 status in the gastric cancer?

Methods: Formalin-fixed, paraffin-embedded sections of 116 pair surgically resected and biopsy specimens as well as 80 pair primary and metastatic lesions of gastric cancer patients were analyzed. Protein expression was assessed using immunohistochemistry $(\mathrm{IHC})$ and graded by the modified scoring criteria for gastric cancer. Gene amplification was evaluated by fluorescence in situ hybridization (FISH) in IHC $2+$ cases.

Results: The positive rate of HER2 was $11.2 \%$ in both surgical and biopsy samples. The consistent rate of HER2 expression was $91.4 \%(106 / 116)$ between biopsy and surgical samples, $P=0.666$. The positive rate of HER2 was $20.5 \%$ in primary and $15.9 \%$ in metastatic samples, $P=0.1876$. The consistent rate of HER2 expression was $90.9 \%(40 / 44)$ between primary and metastatic samples, $\mathrm{P}=0.580$. The consistent rate of Lauren classification was $64.7 \%(75 / 116)$ between biopsy and surgical sample, and $92.5 \%(74 / 80)$ between primary and metastatic samples.

Discussion: For gastric cancer, HER2 expression and Lauren classification were highly homogenous in biopsy and surgical samples, primary and their corresponding metastatic samples. The high concordance observed between these two cohorts indicated that the HER2 examination and Lauren classification of biopsy samples from the primary tumor could well represent the metastatic lesions of the patients.
\end{abstract}

Key words: HER2, Gastric Cancer, Immunohistochemistry, FISH. 


\section{Introduction}

Gastric cancer (GC) is the second most common cause of cancer-related death worldwide [1]. The incidence of gastric carcinoma varies significantly from one part of the world to another and it is particularly common in Eastern Asia, especially in China [2].

Trastuzumab in combination with chemotherapy was approved for treatment of human epidermal growth factor receptor-2 (HER2, also known as ERBB2) positive advanced gastric or gastro-esophageal junction (GEJ) cancer [3]. Amplification, overexpression or both, of HER2, a transmembrane receptor tyrosine kinase, is present in around $6.1-23.0 \%$ of gastric cancers [3-6]. For most advanced GC or GEJ cancer, there was only biopsy sample and no surgical resection sample. We all know that gastric cancer is a heterogeneity disease. Can the HER2 expression in the biopsy sample represent the HER2 status in the surgical sample? Is there heterogeneity for the HER2 expression between the primary and metastatic lesions?

Although the Lauren classification system can date back to 1965, it is still widely accepted and employed by pathologists and physicians today. According to the Lauren classification, gastric adenocarcinomas can be divided into diffuse, intestinal and mixed type [7]. Cohesive cells that form gland-like structures characterize the intestinal type. For the diffuse type, tumor cells lack cell-to-cell interactions and infiltrate the stroma as single cell or small subgroups, leading to a population of non-cohesive, scattered tumor cells [7]. The intestinal-type is more frequent in males and in elderly patients, while the diffuse-type occurs more frequently in women and young patients [8]. Intestinal type patients have better outcomes than those with diffuse type [6, 8-11]. While HER2 positivity is more common in intestinal type gastric cancer [12]. Are the Lauren classification consistent between biopsy and surgical samples, primary and metastatic lesions?

In the present study, we try to answer the questions above by analyzing the Lauren classification and HER2 expression between biopsy and surgical samples, primary and metastatic lesions in gastric cancer patients.

\section{Materials and Methods}

\section{Patient collection}

\section{Cohort 1: Biopsy and surgical resection samples}

From January 2014 to December 2015, we collected clinical information retrospectively from gastric cancer patients who received both biopsy and surgical resection in our cancer center. All patients were categorized according to the $7^{\text {th }}$ American Joint Committee on Cancer (AJCC) Tumor-Node-Metastasis (TNM) stage. Patients included in the study met the following criteria: (1) histologically confirmed gastric adenocarcinoma patients; (2) adequate paraffin-embedded tumor tissue samples for Lauren classification analysis and HER2 examination; (3) patients received both biopsy under gastroscopy and gastrectomy; (4) full medical records with regular survival follow-up data. The exclusion criteria were: (1) age <18 years old; and (2) other malignancy within the last 5 years, except carcinoma in situ of the cervix, or basal cell carcinoma.

\section{Cohort 2: Primary and metastatic lesion samples}

From January 2000 to December 2013, we collected clinical information retrospectively from gastric cancer patients who received both biopsy of primary and metastatic lesion. Patients included in the study met the following criteria: (1) histologically confirmed gastric adenocarcinoma patients; (2) AJCC TNM $7^{\text {th }}$ stage IV; (3) received biopsy from both primary and metastatic lesions; (4) full medical records with regular survival follow-up data. The exclusion criteria were: (1) age $<18$ years old; and (2) other malignancy within the last 5 years, except carcinoma in situ of the cervix, or basal cell carcinoma.

\section{Lauren classification}

Assignment of histological type was based on the Lauren criteria. The intestinal type was described as a tumor with glandular architecture, resembling colonic carcinoma. The diffuse type was described as a tumor composed of solitary or small clusters of cells, and lacking glandular structures. The mixed type was described as the combination of these two features. Two pathologists reviewed the original diagnostic slides to make a diagnosis of Lauren classification.

\section{HER2 evaluation}

\section{Immunohistochemistry (IHC)}

For all patients, HER2 expression was detected by IHC. IHC staining was carried out using an anti-HER-2/NEU (4B5) antibody (Ventana Medical Systems, Inc. Tucson, AZ, USA) against HER2 on a Ventana Benchmark XT automatic staining system, according to the manufacturer's instructions. The amended HER2 IHC scoring system for gastric cancer proposed by Hoffmann et al. was used as the criteria for scoring [13]. Examples of IHC expression from negative to,+++ and +++ were shown in figure 1 . 

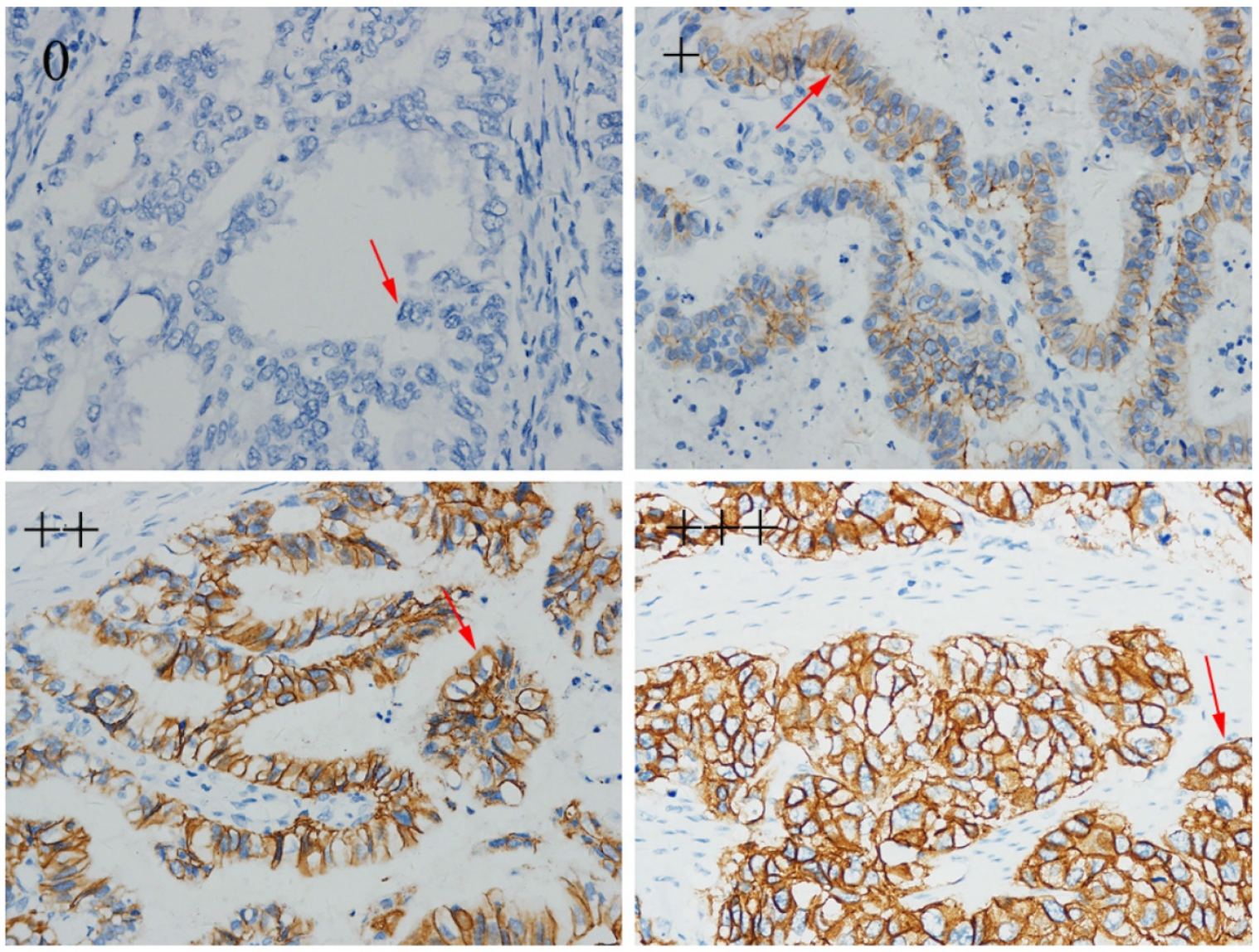

Figure 1. Human epidermal growth factor receptor 2 (HER2) status by immunohistochemistry (magnification $\times 400)$.

\section{Fluorescence in situ hybridization (FISH)}

HER2 amplification levels were measured when the result of IHC was 2+. The PathVysion ${ }^{\circledR} H E R 2$ DNA Probe kit (LSI®HER2/neu Spectrum Orange ${ }^{\mathrm{TM}} /$ chromosome 7 centromere probe (CEP) ®17 Spectrum Green) was used for FISH analysis, according to the manufacturer's protocol. A positive result from FISH was defined as a HER2:CEP17 ratio $\geq 2$. Example of HER2 FISH positive and negative were shown in figure 2. Any case with IHC $3+$ or IHC2+/FISH + was considered as HER2-positive, while cases with IHC 0 or IHC $1+$ or IHC $2+$ /FISH were considered as HER2- negative, according to criteria of the European Medicines Agency.

\section{Statistical analysis}

The patients' demographic and tumor characteristics were summarized with descriptive statistics. Comparisons of categorical variables between biopsy and surgical resection lesions or primary and metastatic diseases were performed using the Chi square test, and continuous variables were compared using Student's t test. All of statistical analyses were performed using the Intercooled Stata
13.0 (Stata Corporation, College Station, TX). Statistical significance was set at two-sided $P<0.05$.

\section{Informed consent}

All patients signed written informed consent for their information to be used for the study. The independent ethics committees at the Cancer Center of Sun Yat-sen University approved the study. The study was undertaken in accordance with the ethical standard of the World Medical Association Declaration of Helsinki.

\section{Results}

\section{Cohort 1. Biopsy and surgical resection samples}

\section{Patient demographics}

There were 116 patients with pair samples (Table 1). The median age was 59 years (range: 29 to 78 years old); 80 were male and 36 were female. There were 20 stage IV patients at the time of diagnosis who received gastrectomy to relieve the symptom of obstruction or bleeding. Until December 1, 2016, 8 patients died from gastric cancer. 

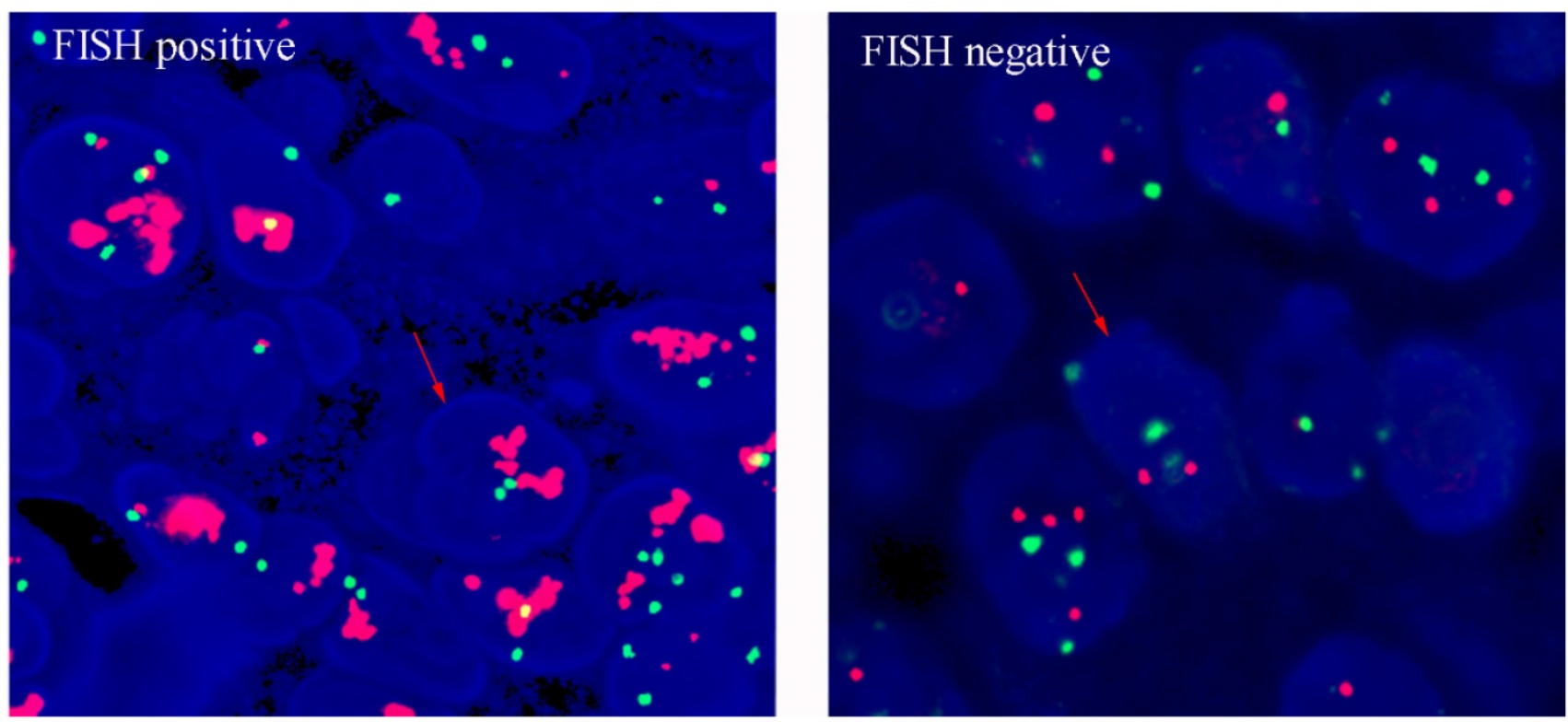

Figure 2. Human epidermal growth factor receptor 2 (HER2) status by FISH (magnification×400).

\section{Lauren classification}

There were $44.0 \%(51 / 116)$ of patients with intestinal type and $36.2 \%(42 / 116)$ patients with diffuse type for the biopsy samples. For the surgical samples, $40.5 \%(47 / 116)$ of the patients were intestinal type and $32.8 \%(38 / 116)$ were diffuse type. The consistent rate of Lauren classification between biopsy and surgical sample was $64.7 \%$ (75/116), $P=0.461$ (Table 2).

\section{HER2 expression}

Table 3 showed the detailed distribution of HER2 IHC expression between biopsy and surgical samples. According to the IHC result, the consistent rate of HER2 IHC expression between biopsy and surgical samples was $62.9 \%(73 / 116)$. Thirteen biopsy samples and 15 surgical samples were IHC 2+ for HER2. FISH was carried out in these samples and we found that 7 biopsy samples and 6 surgical samples were FISH positive. The positive rate of HER2 was $11.2 \%$ in both surgical and biopsy samples. The consistent rate of HER2 expression was 91.4\% $(106 / 116)$ between biopsy and surgical samples, $P=0.666$. The 10 patients who showed discordance between the biopsy and surgical samples included 4 biopsy positive, surgical negative and 6 biopsy negative while surgical positive.

\section{Cohort 2. Primary and metastatic samples}

\section{Patient demographics}

There were 80 patients with pair samples (Table 4). The median age was 58 years (range: 28 to 84 years old); 54 were male and 26 were female. The most common metastatic site was peritoneal lesion. Until December 1, 2016, 47 patients died from gastric cancer.

Table 1. Demographic feature of the 116 pair gastric cancer patients of biopsy and surgical samples.

\begin{tabular}{|c|c|c|}
\hline & Number of patients (N=116) & Percentage (\%) \\
\hline \multicolumn{3}{|l|}{ Gender } \\
\hline Female & 36 & 31.03 \\
\hline Male & 80 & 68.97 \\
\hline Age (Mean $\pm S D)$ & $57.07 \pm 11.86$ & \\
\hline \multicolumn{3}{|l|}{ Primary site } \\
\hline Gastric & 92 & 79.31 \\
\hline Gastroesophageal junction & 24 & 20.69 \\
\hline \multicolumn{3}{|l|}{ Degree of differentiation } \\
\hline High to moderate poor & 38 & 32.76 \\
\hline Poor and signet ring cell & 78 & 67.24 \\
\hline \multicolumn{3}{|l|}{ TNM stage } \\
\hline I & 16 & 13.79 \\
\hline II & 31 & 26.72 \\
\hline III & 49 & 42.24 \\
\hline IV & 20 & 17.24 \\
\hline \multicolumn{3}{|l|}{ T stage } \\
\hline $\mathrm{T} 1$ & 15 & 12.93 \\
\hline $\mathrm{T} 2$ & 18 & 15.52 \\
\hline $\mathrm{T} 3$ & 50 & 43.10 \\
\hline $\mathrm{T} 4$ & 33 & 28.45 \\
\hline \multicolumn{3}{|l|}{$\mathrm{N}$ stage } \\
\hline No & 27 & 23.28 \\
\hline N1 & 17 & 14.66 \\
\hline N2 & 23 & 19.82 \\
\hline N3 & 49 & 42.24 \\
\hline \multicolumn{3}{|l|}{ M stage } \\
\hline M0 & 96 & 82.76 \\
\hline M1 & 20 & 17.24 \\
\hline \multicolumn{3}{|l|}{ Neurolymphatic invasion } \\
\hline No & 36 & 31.03 \\
\hline Yes & 80 & 68.97 \\
\hline Tumor size $($ Mean \pm SD) $\mathrm{cm}$ & $4.45 \pm 2.75$ & \\
\hline
\end{tabular}




\section{Lauren classification}

There were $55.0 \%(44 / 80)$ of patients with intestinal type and $40.0 \%(32 / 80)$ patients with diffuse type in the primary samples. For the metastatic samples, $57.5 \%(46 / 80)$ of the patients were intestinal type and $37.5 \%(30 / 80)$ were diffuse type. The consistent rate of Lauren classification between primary and metastatic sample was $92.5 \%$ (74/80), $P=0.947$ (Table 2).

Table 2. Comparison of Lauren classification in pair samples of gastric cancer patients

\begin{tabular}{lllll}
\hline & & Biopsy sample & & \\
Intestinal & Diffuse & Mixed \\
Surgical & Intestinal & 36 & 8 & 3 \\
sample & Diffuse & 7 & 25 & 6 \\
& Mixed & 8 & 9 & 14 \\
& & Primary sample & \\
Metastatic & Intestinal & 43 & Diffuse & Mixed \\
samples & Diffuse & 0 & 2 & 1 \\
& Mixed & 1 & 29 & 1 \\
& & 1 & 2 \\
\hline
\end{tabular}

\section{HER2 status}

Only 44 samples have enough tissues for the IHC examination of HER2. Table 3 showed the detailed distribution of HER2 IHC expression between primary and metastatic samples. According to the IHC result, the consistent rate of HER2 IHC expression between primary and metastatic samples was $81.8 \%(36 / 44)$. Five primary samples and 3 metastatic samples were IHC 2+ for HER2. FISH was carried out in these samples and we found that 4 primary samples and 3 metastatic samples were FISH positive. The positive rate of HER2 was $20.5 \%$ in primary and $15.9 \%$ in metastatic samples, $P=0.1876$. The consistent rate of HER2 expression was $90.9 \%$ $(40 / 44)$ between primary and metastatic samples, $P=0.580$. Only 4 patients were discordant between the primary and metastatic samples including 3 primary positive, metastatic negative and 1 primary negative, metastatic positive patient.

Table 3. Comparison of human epidermal growth factor receptor 2 (HER2) status in pair samples of gastric cancer patients

\begin{tabular}{llllll}
\hline \multirow{5}{*}{ Surgical } & 0 & 0 & \multicolumn{4}{l}{ Biopsy sample } & ++ & +++ \\
sample & + & 55 & 11 & 2 & 0 \\
& ++ & 18 & 6 & 2 & 0 \\
& +++ & 5 & 2 & 7 & 1 \\
& & 0 & 0 & 2 & 5 \\
& & Primary sample & & \\
Metastatic & 0 & 0 & + & ++ & +++ \\
samples & + & 28 & 1 & 1 & 1 \\
& ++ & 1 & 2 & 2 & 0 \\
& +++ & 0 & 0 & 2 & 0 \\
& ++ & 0 & 0 & 4
\end{tabular}

Table 4. Demographic feature of the 80 pair gastric cancer patients of primary and metastatic samples

\begin{tabular}{lll}
\hline & $\begin{array}{l}\text { Number of patients } \\
(\mathrm{N}=80)\end{array}$ & Percentage (\%) \\
\hline Gender & 26 & 32.5 \\
Female & 54 & 67.5 \\
Male & $58.2(11.49)$ & \\
Age (Mean \pm SD) & & \\
Primary site & 15 & 18.8 \\
Gastric & 65 & 81.3 \\
Gastroesophageal junction & & \\
Degree of differentiation & & 21.3 \\
High to moderate poor & 17 & 78.8 \\
Poor and signet ring cell & 63 & \\
Metastasis sites & & 42.5 \\
Liver & 34 & 7.5 \\
Lung & 6 & 46.3 \\
Peritoneal nodules & 37 & 3.8 \\
Others & 3 & \\
\hline
\end{tabular}

\section{Discussion}

The result of the phase III ToGA trial indicated trastuzumab plus cisplatin and fluoropyrimidine chemotherapy as the standard care for patients with HER2-positive advanced gastric cancer [3]. Based on this study, patients with advanced or metastatic adenocarcinoma of the stomach or esophagogastric junction for whom trastuzumab therapy is being considered, assessment for tumor HER2 overexpression using IHC is recommended. The HER2 status in advanced gastric carcinoma is usually assessed on the biopsy samples from the primary tumor because patients with metastatic disease rarely undergo surgery or biopsy from the metastatic disease. Can the HER2 status of the biopsy sample from the primary lesion truly represent the HER2 status in the gastric cancer? The issue can be further divided into 2 questions: the concordance of HER2 status between biopsy and surgical samples, between primary tumor and metastatic lesions.

Concordance of HER2 status between primary tumor and metastasis has been occasionally addressed in the literatures and the results are controversial. Bozzetti $\mathrm{C}$ et al. found that concordance of FISH results was $98.5 \%$ in 68 primary and matched metastatic sites and concordance of IHC results was $94.9 \%$ in 39 paired cases [14]. Marx AH et al. also showed that HER-2 status was identical in primary and their matched lymph node metastases [15]. Kochi et al. demonstrated a HER 2 concordance rate of $90.2 \%$ between the primary gastric and the corresponding lymph node metastasis in 102 patients [16]. While Gumusay $\mathrm{O}$ et al. reported that $16.2 \%$ of the patients had a HER2 discordance between primary lesion and the paired metastatic lesion as assessed by the SISH method [17]. Cho et al. found that discrepancies in HER2 status between primary and paired metastatic 
lymph nodes were $11 \%$ by IHC, $7 \%$ by SISH [18]. Peng $\mathrm{Z}$ et al. made a meta-analysis using 1,867 patients from eighteen articles and found that pooled discordance proportions between primary and metastasis lesions were $7 \%$ [95\% confidence interval (CI): $5-10 \%$ ] for HER2 status [19]. Pooled proportions of tumors shifting from positive to negative and from negative to positive were $17 \%$ (95\% CI: $7-29 \%$ ) and $4 \%$ (95\% CI: 2-6\%) respectively. In another study, HER2 positive was more common in liver metastasis than in the paired lymph nodes [20]. We found that the consistent rate of HER2 expression was $90.9 \%(40 / 44)$ between primary and metastatic samples by combining IHC and FISH examination. The high concordance rate between primary and metastatic samples indicated that in gastric cancer HER2 status remained unchanged in most cases during the metastatic process. Since all the patients in our study were synchronous metastasis, we had no idea whether the HER2 status kept unchanged for the metachronous metastasis. While for synchronous metastasis, it is not necessary to test the HER2 status from both primary and metastatic lesions basing on our results.

Concordance of HER2 status between biopsy tumor and surgical tumor has been scarcely addressed. Intratumoral heterogeneity of gastric cancer was a major challenge in HER2 biopsy samples. Kanayama $\mathrm{K}$ et al. found that the overall concordance rate of HER2 status between biopsy tissues and surgical specimens was 91.4\% [21]. However, in another study, Yoshida $\mathrm{H}$ et al. showed that the concordance rate of IHC results between surgically resected specimens and the corresponding biopsy specimens was only $57.0 \%$, and in discordant cases, HER2 positivity in biopsies and HER2 negativity in surgically resected tumors were most common [22]. Biopsy from multiple sites was encouraged to decrease the bias caused by the intratumoral heterogeneity [23]. In our study, we found that the consistent rate of HER2 expression was $91.4 \%(106 / 116)$ between one-point biopsy and surgical samples. For the biopsy samples, we only carried out the HER2 test in one of the biopsies.

In this paper, we analyzed the Lauren classification between the biopsy and surgical samples as well as the primary and metastatic samples. We found that the consistent rate of Lauren classification was $64.7 \%(75 / 116)$ and $92.5 \%(74 / 80)$ between biopsy and surgical sample, primary and metastatic samples, respectively. For the biopsy and surgical paired samples, most of the discordance had mixed type and only 15 patients (12.9\%) had totally different Lauren classification between the biopsy and surgical samples, one diffuse type and the other intestinal type. We did not find other reports about the concordance rate of Lauren classification in different samples of gastric cancer in the literatures.

In all, we found that HER2 expression was highly homogenous in gastric cancer. Identical HER2 status was found in biopsy and surgical samples, primary and their corresponding metastatic samples. The high concordance observed between these two cohorts indicated that the HER2 examination of biopsies from the primary tumor could well represent the HER2 status of the patients. The concordance rate of Lauren classification was higher between the primary and metastatic samples than between the biopsy and surgical samples.

Potential limitation of current study is its retrospective methodology from a single-institution experience. Moreover, we did not collect metachronous metastasis samples, therefore, we have no idea about the heterogeneity in metachronous metastatic diseases.

\section{Acknowledgments}

This work was supported by National Natural Science Foundation of China (Grant number. 81602066); the Fundamental Research Funds for the Central Universities (Grant number. 16ykpy25); the third outstanding young talents training plan and Medical Scientist program of Sun Yat-sen University cancer center; Medical Scientific Research of Guangdong province B2014161; Science and Technology Planning Project of Guangdong Province, China (Grant number. 2013A022100023); Natural Science Foundation of Guangdong Province, China (Grant number.2014A030312015).

\section{Competing Interests}

The authors have declared that no competing interest exists.

\section{References}

1. Kamangar F, Dores GM, Anderson WF. Patterns of cancer incidence, mortality, and prevalence across five continents: defining priorities to reduce cancer disparities in different geographic regions of the world. Journal of clinical oncology : official journal of the American Society of Clinical Oncology. 2006; 24: 2137-50.

2. Chen $\mathrm{W}$, Zheng $\mathrm{R}$, Zeng $\mathrm{H}$, Zhang $\mathrm{S}$. The updated incidences and mortalities of major cancers in China, 2011. Chinese journal of cancer. 2015; 34: 502-7.

3. Bang YJ, Van Cutsem E, Feyereislova A, Chung HC, Shen L, Sawaki A, et al. Trastuzumab in combination with chemotherapy versus chemotherapy alone for treatment of HER2-positive advanced gastric or gastro-oesophageal junction cancer (ToGA): a phase 3, open-label, randomised controlled trial. Lancet (London, England). 2010; 376: 687-97.

4. Hsu JT, Chen TC, Tseng JH, Chiu CT, Liu KH, Yeh CN, et al. Impact of HER-2 overexpression/amplification on the prognosis of gastric cancer patients undergoing resection: a single-center study of 1,036 patients. The oncologist. 2011; 16: 1706-13.

5. Sheng WQ Huang D, Ying JM, Lu N, Wu HM, Liu YH, et al. HER2 status in gastric cancers: a retrospective analysis from four Chinese representative clinical centers and assessment of its prognostic significance. Annals of oncology : official journal of the European Society for Medical Oncology. 2013; 24: $2360-4$

6. Qiu M, Zhou Y, Zhang X, Wang Z, Wang F, Shao J, et al. Lauren classification combined with HER2 status is a better prognostic factor in Chinese gastric cancer patients. BMC cancer. 2014; 14: 823. 
7. Lauren P. THE TWO HISTOLOGICAL MAIN TYPES OF GASTRIC CARCINOMA: DIFFUSE AND SO-CALLED INTESTINAL-TYPE CARCINOMA. AN ATTEMPT AT A HISTO-CLINICAL CLASSIFICATION. Acta pathologica et microbiologica Scandinavica. 1965; 64: 31-49.

8. Qiu MZ, Cai MY, Zhang DS, Wang ZQ, Wang DS, Li YH, et al. Clinicopathological characteristics and prognostic analysis of Lauren classification in gastric adenocarcinoma in China. Journal of translational medicine. 2013; 11: 58

9. Tanner M, Hollmen M, Junttila TT, Kapanen AI, Tommola S, Soini Y, et al. Amplification of HER-2 in gastric carcinoma: association with Topoisomerase IIalpha gene amplification, intestinal type, poor prognosis and sensitivity to trastuzumab. Annals of oncology : official journal of the European Society for Medical Oncology. 2005; 16: 273-8.

10. Yamashita K, Sakuramoto S, Katada N, Futawatari N, Moriya H, Hirai K, et al. Diffuse type advanced gastric cancer showing dismal prognosis is characterized by deeper invasion and emerging peritoneal cancer cell: the latest comparative study to intestinal advanced gastric cancer. Hepato-gastroenterology. 2009; 56: 276-81.

11. Zheng H, Takahashi H, Murai $\mathrm{Y}$, Cui Z, Nomoto K, Miwa S, et al. Pathobiological characteristics of intestinal and diffuse-type gastric carcinoma in Japan: an immunostaining study on the tissue microarray. Journal of clinical pathology. 2007; 60: 273-7.

12. Qiu MZ, Li Q, Wang ZQ, Liu TS, Liu Q, Wei XL, et al. HER2-positive patients receiving trastuzumab treatment have a comparable prognosis with HER2-negative advanced gastric cancer patients: a prospective cohort observation. International journal of cancer. 2014; 134: 2468-77.

13. Gianni L, Eiermann W, Semiglazov V, Manikhas A, Lluch A, Tjulandin S, et al. Neoadjuvant chemotherapy with trastuzumab followed by adjuvant trastuzumab versus neoadjuvant chemotherapy alone, in patients with HER2-positive locally advanced breast cancer (the NOAH trial): a randomised controlled superiority trial with a parallel HER2-negative cohort. Lancet (London, England). 2010; 375: 377-84.

14. Bozzetti C, Negri FV, Lagrasta CA, Crafa P, Bassano C, Tamagnini I, et al. Comparison of HER2 status in primary and paired metastatic sites of gastric carcinoma. British journal of cancer. 2011; 104: 1372-6.

15. Marx AH, Tharun L, Muth J, Dancau AM, Simon R, Yekebas E, et al. HER-2 amplification is highly homogenous in gastric cancer. Human pathology. 2009; 40: 769-77.

16. Kochi M, Fujii M, Masuda S, Kanamori N, Mihara Y, Funada T, et al. Differing deregulation of HER2 in primary gastric cancer and synchronous related metastatic lymph nodes. Diagnostic pathology. 2013; 8: 191

17. Gumusay O, Benekli M, Ekinci O, Baykara M, Ozet A, Coskun U, et al. Discordances in HER2 status between primary gastric cancer and corresponding metastatic sites. Japanese journal of clinical oncology. 2015; 45: 416-21.

18. Cho EY, Park K, Do I, Cho J, Kim J, Lee J, et al. Heterogeneity of ERBB2 in gastric carcinomas: a study of tissue microarray and matched primary and metastatic carcinomas. Modern pathology : an official journal of the United States and Canadian Academy of Pathology, Inc. 2013; 26: 677-84.

19. Peng Z, Zou J, Zhang X, Yang Y, Gao J, Li Y, et al. HER2 discordance between paired primary gastric cancer and metastasis: a meta-analysis. Chinese journal of cancer research. 2015; 27: 163-71.

20. Wei Q, Xu J, Shen L, Fu X, Zhang B, Zhou X, et al. HER2 expression in primary gastric cancers and paired synchronous lymph node and liver metastases. A possible road to target HER2 with radionuclides. Tumour biology : the journal of the International Society for Oncodevelopmental Biology and Medicine. 2014; 35: 6319-26.

21. Kanayama K, Imai H, Yoneda M, Hirokawa YS, Shiraishi T. Significant intratumoral heterogeneity of human epidermal growth factor receptor 2 status in gastric cancer: A comparative study of immunohistochemistry, FISH, and dual-color in situ hybridization. Cancer science. 2016; 107: 536-42.

22. Yoshida $\mathrm{H}$, Yamamoto $\mathrm{N}$, Taniguchi $\mathrm{H}$, Oda I, Katai $\mathrm{H}$, Kushima $\mathrm{R}$, et al. Comparison of HER2 status between surgically resected specimens and matched biopsy specimens of gastric intestinal-type adenocarcinoma. Virchows Archiv : an international journal of pathology. 2014; 465: 145-54.

23. Kubo A, Corley DA. Marked regional variation in adenocarcinomas of the esophagus and the gastric cardia in the United States. Cancer. 2002; 95: 2096-102 